\title{
Changes in membrane lipids and carotenoids during light acclimation in a marine cyanobacterium Synechococcus sp.
}

\author{
Olimpio Montero ${ }^{1 *}$, Alberto SÁnchez-Guijo ${ }^{2}$, Luis M Lubián ${ }^{3}$ \\ and Gonzalo Martínez-RodríGuez ${ }^{3}$ \\ ${ }^{1}$ Centre for Biotechnology Development (CDB), CSIC, Francisco Vallés 8, 47151 \\ Boecillo (Technological Park), Valladolid, Spain \\ ${ }^{2}$ Institute of Molecular Biology and Genetics (IBGM), CSIC-UVA, Sanz y Fores 3, 47003 \\ Valladolid, Spain \\ ${ }^{3}$ Institute for Marine Sciences of Andalusia (ICMAN), CSIC, Av. República Saharaui 2, \\ 11510 Puerto Real, Cádiz, Spain \\ *Corresponding author (Fax, +34-983345016; Email, olimpio.montero@dicyl.csic.es)
}

\begin{abstract}
Time course of carotenoid and membrane lipid variation during high light (HL) acclimation (about $85 \mu \mathrm{mol} \mathrm{m}^{-2} \mathrm{~s}^{-1}$ ), after transfer from low light (LL) $\left(5-10 \mu \mathrm{mol} \mathrm{m}{ }^{-2} \mathrm{~s}^{-1}\right)$, was determined in a marine Synechococcus strain. Highperformance liquid chromatography (HPLC) coupled to diode array detector (DAD) or electrospray ionization mass spectrometry (ESI-MS) was used for compound separation and detection. Myxoxanthophyll rose within a time interval of $8 \mathrm{~h}$ to $24 \mathrm{~h}$ after the onset of exposure to HL. $\beta$-carotene content started to decrease after $4 \mathrm{~h}$ of the onset of exposure to HL. Zeaxanthin content rose with exposure to HL, but it was only significant after $24 \mathrm{~h}$ of exposure. Carotenoid changes are in agreement with a coordinated activity of the enzymes of the myxoxanthophyll biosynthetic pathway, with no rate-limiting intermediate steps. Lipid analysis showed all species with a C18:3/C16:0 composition increased their content, the changes of $\mathrm{PG}(18: 3 / 16: 0)$ and MGDG(18:3/16:0) being primarily significant. Major lipid changes were also found to occur within $24 \mathrm{~h}$. These changes might suggest reduction and reorganization of the thylakoid membrane structure. Hypotheses are also drawn on the role played by lipid molecule shape and their possible effect in membrane fluidity and protein accommodation.
\end{abstract}

[Montero O, Sánchez-Guijo A, Lubián LM and Martínez-Rodríguez G 2012 Changes in membrane lipids and carotenoids during light acclimation in a marine cyanobacterium Synechococcus sp. J. Biosci. 37 635-645] DOI 10.1007/s12038-012-9234-2

\section{Introduction}

Light acclimation of cyanobacterial cells is a process that involves a reduction of chlorophyll and phycobiliprotein contents, besides a change in the relative proportion of carotenoids (Riethman et al. 1988; Hirschberg and Chamovitz 1994; Falkowsky and Raven 1997; McIntyre et al. 2002; Schagerl and Müller 2006). In Synechocystis strains, the increase in the glycocarotenoid Myx that is elicited by high light exposure has been explained by the need for protection against
ROS that are generated in the photosynthetic light reactions (Lagarde and Vermaas 1999; Steiger et al. 1999; Schäfer et al. 2005). The rise in Myx is also observed in some Synechococcus strains under high light or nitrogen deficiency (Masamoto and Furukawa 1997; Graham and Bryant 2008; Montero et al. 2011). A biological function for myxoxanthophyll alternative to that of photoprotection has been suggested by Mohamed et al. (2005). Using a mutant of Synechocystis sp. strain PCC6803 that lacks fucose synthetase activity and, thereby, myxoxanthophyll, a functional

Keywords. Carotenoids; galactosyl-diacyl-glycerolipids; HPLC-MS; phosphatidylglycerol; Synechococcus sp.

Abbreviations used: Car, $\beta$-carotene; Chl, chlorophyll $a$; HL, high light; LL, low light; Lyc, lycopene; MGDG and DGDG, mono- and digalactosyl-diacyl-glycerol; Myx, myxoxanthophyll (myxol-2'-fucoside); PG, phosphatidyl-diacyl-glycerol; PRCs, photosynthetic reaction centers; SQDG, sulfoquinovosyl-diacyl-glycerol; Z, zeaxanthin 
role for myxoxanthophyll was proposed by these authors in relation to thylakoid membrane stabilization and S-layer formation. Nonetheless, experimental evidence available till date does not clearly highlight on the actual biological function of this glycocarotenoid.

Recent research has unraveled the myxoxanthophyll biosynthetic pathway almost completely (Graham and Bryant 2009). The involvement of the same $\beta$-carotene hydroxylase (crtR gene) that participates in $\mathrm{Z}$ biosynthesis through 3hydroxylation was early demonstrated by Lagarde and Vermaas (1999). The Myx biosynthetic pathway proposed by Graham and Bryant (2009) encompasses 1'-hydroxylation of Lyc as the first step (CruF enzyme) (figure 1); thereby, since Car is formed through two cyclization steps from Lyc, a competition for the Lyc pool is likely to arise between the two pathways. Furthermore, the $\mathrm{Z}$ content is also dependent on the Car pool that is available for the $\beta$-carotene hydroxylase enzyme. Hence, it is expected high light acclimation to involve a transition interval during which the cellular contents of $\mathrm{Car}$ and $\mathrm{Z}$ are reduced. In particular, since $\mathrm{Z}$ has been reported to increase with exposure to high light in Cyanobacteria (Masamoto and Furukawa 1997), Lyc content should also increase to satisfy the demand from the two pathways. To our knowledge, there is not report yet on the time course of Myx rise and other carotenoid variation during light acclimation in vivo.

Membrane lipids have long been regarded as having a structural function only, but recent research is pointing out

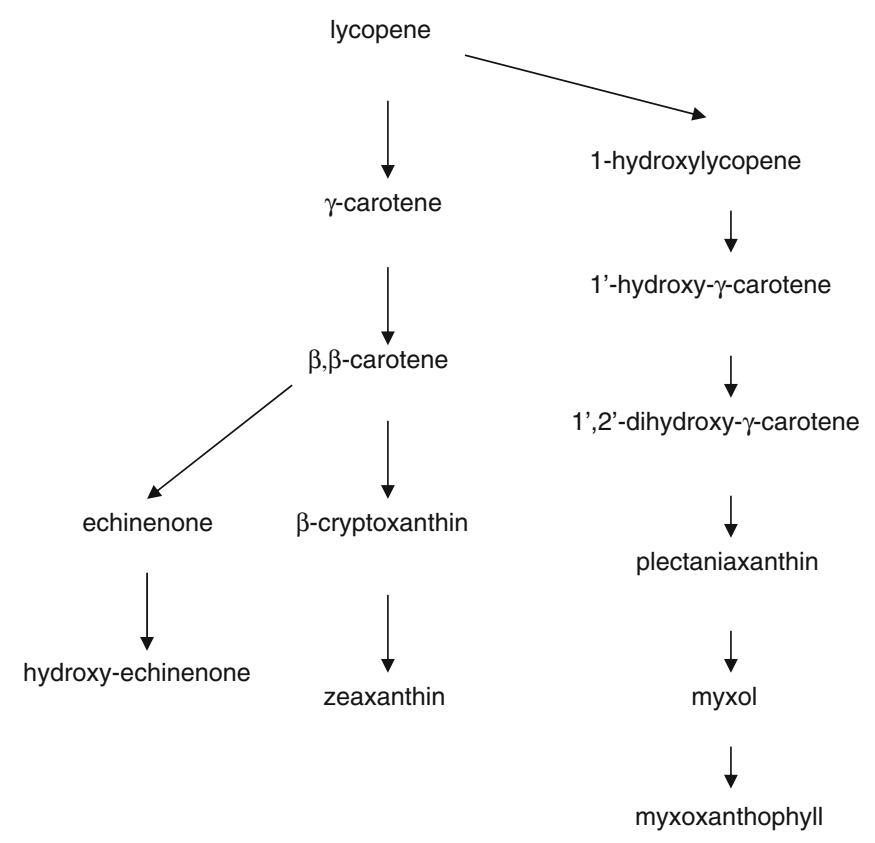

Figure 1. Biosynthetic pathways for echinenone, zeaxanthin and myxoxanthophyll from lycopene in cyanobacteria, according to Lagarde and Vermaas (1999) and Graham and Bryant (2009). lipids are involved in different physiological processes in a specific manner, which accounts for interaction with membrane proteins and participation in different membrane functions (Lee 2004; Palsdottir and Hunte 2004; McIntosh and Simon 2006; Jones 2007; Hölzl and Dörmann 2007). Cyanobacterial membrane lipids have shown changes in their contents that relied on environmental factors like temperature, light, salt stress and drought (Riethman et al. 1988; Sakamoto et al. 1997; Hölzl and Dörmann 2007; Sato and Wada 2009). The lipid profile of cyanobacterial membranes is composed of mono- and di-galactosyl-diacyl-glycerol (MGDG and DGDG, respectively), sulfoquinovosyl-diacylglycerol (SQDG), and phosphatidyl-diacyl-glycerol (PG). C18 chains are preferred at the $s n-1$ position of the glycerol backbone, while $\mathrm{C} 16$ chains are preferred at the $s n-2$ position (Zepke et al. 1978; Hölzl and Dörmann 2007; Sato and Wada 2009). An essential role for PG has been found in photosynthesis (Sato et al. 2000; Domonkos et al. 2004; Sato 2004; Wada and Murata 2007) and regulation of carotenoid biosynthesis (Domonkos et al. 2009); SQDG and/or DGDG seem to substitute PG under phosphate limitation (Güler et al. 1996; Dörmann and Benning 2002; Sato 2004; Frentzen 2004; Awai et al. 2007; Kobayashi et al. 2009); and MGDG or the MGDG/DGDG ratio has been bound to the upkeep of the PSII complex functional configuration and efficient energy transfer between the photosystem II antenna complexes and core complex (Grzyb et al. 2008; Leng et al. 2008; Zhou et al. 2009).

Till date, changes in lipid composition have been primarily determined by gas chromatography coupled to electron impact mass spectrometry (GC-EI-MS), with separation of the different lipid classes by thin-layer chromatography and further fatty acid saponification with $\mathrm{KOH}$ in methanol (methylation). This procedure imposes some constrains to the ascertaining of glycerolipid variations at the species level, which may be avoided by analysis with mass spectrometry techniques as they are implemented at present, namely, electrospray ionization coupled to ion-trap or triple-quadrupole analysers (Welti et al. 2003; Yao et al. 2006; Okazaki et al. 2009). A highperformance liquid chromatography coupled to mass spectrometry (HPLC-MS) method was used in this study that enabled lipid isobaric species separation as well as ascertaining their content time course during light acclimation. Time course variation of carotenoids was simultaneously determined using HPLC-DAD measurements.

\section{Material and methods}

\subsection{Culture and experimental setup}

The Synechococcus sp. strain 01/0202 from the Marine Microalgae Culture Collection of the Institute for Marine Sciences of Andalusia (ICMAN, CSIC) was used in this 
study. This strain was previously found to be closely related to the model strain Synechococcus sp. PCC7002 according to $16 \mathrm{~S}$ rDNA, although lacking the carotenoid synechoxanthin (Montero et al. 2011). Cells were grown in $100 \mathrm{~mL}$ erlenmeyer flasks containing $60 \mathrm{~mL}$ of culture volume, and were placed in a thermostated culture chamber (Friocell-111, MMM Medcenter Einrichtungen GmbH, Gräfelging/München, Germany). Light was provided by daylight fluorescent tubes. Culture medium was natural seawater filtered through $1.0 \mu \mathrm{m}$ filter, sterilized in an autoclave $\left(120^{\circ} \mathrm{C}, 1 \mathrm{~kg}\right.$ pressure, $60 \mathrm{~min}$ ), and enriched with $\mathrm{F} / 2$ medium with double nitrate and phosphate concentrations. Culture temperature was $20^{\circ} \mathrm{C}$, and irradiance was 5-10 $\mu \mathrm{mol}$ photons $\mathrm{m}^{-2} \mathrm{~s}^{-1}$ for LL conditions and $85-90 \mu \mathrm{mol}$ photons $\mathrm{m}^{-2} \mathrm{~s}^{-1}$ for HL conditions. These irradiances were chosen because previous experiments on growth pointed out there was a significant difference between both irradiances regarding growth but without stress.

For experiments, cultures were freshly prepared by adding 5 or $10 \mathrm{~mL}$ of the stock culture to autoclaved seawater with $\mathrm{F} / 2$ medium, and kept at low irradiance for $48 \mathrm{~h}$ in order to get the cells acclimated again to the LL conditions. Following, the cultures were moved to high light (HL conditions, time 0 ), and $2 \mathrm{~mL}$ samples from 3 independent cultures taken at different time intervals. Cell density was determined by measuring the absorbance of $1 \mathrm{~mL}$ samples at $750 \mathrm{~nm}\left(\mathrm{~A}_{750}\right)$ (Sorokin 1973; Montero et al. 2002); and stock cultures used for inoculums were diluted to give an absorbance (r.u.) between 0.1 and 0.2 when appropriate. Two independent experiments were conducted with each culture in triplicate.

\subsection{Sample preparation}

After a $2 \mathrm{~mL}$ aliquot of culture was taken, $100 \mu \mathrm{L}$ of $4 \%$ paraformaldehide was added to each culture sample, and the samples were placed in ice. Cells were pelleted by centrifugation at $3500 \mathrm{rpm}(2818 \mathrm{~g})$ for $10 \mathrm{~min}$ at $4^{\circ} \mathrm{C}$, and then the supernatant was discarded and $1 \mathrm{~mL}$ of cold $0.9 \%$ ammonium formate was added to the pellet for salt removal, and the suspension centrifuged again. Then $1 \mathrm{~mL}$ of methanol was added to the pellet after the new supernatant was discarded while the sample container was kept in ice. Following, the methanol suspension was sonicated for 1 min while being kept in ice. The methanolic extract was collected after a new centrifugation, and kept at $-20^{\circ} \mathrm{C}$ under nitrogen atmosphere until analysis.

\subsection{Pigment chromatography}

HPLC was used for pigment determination (Montero et al. 2005). Briefly, gradient elution was based on two solvent mixtures that were: (A) methanol : water $(8: 2 \mathrm{v} / \mathrm{v})$ and (B) methanol : acetone (1:1); and the elution sequence was time $0 \min 75 \% \mathrm{~A}+25 \% \mathrm{~B}$, time $4.5 \min 25 \% \mathrm{~A}+75 \% \mathrm{~B}$, time $7.0 \min 25 \% \mathrm{~A}+75 \% \mathrm{~B}$, time $12.0 \min 10 \% \mathrm{~A}+90 \% \mathrm{~B}$, time $15 \mathrm{~min} 100 \% \mathrm{~B}$, time $17 \mathrm{~min} 100 \% \mathrm{~B}$, time $22.0 \mathrm{~min} 75 \% \mathrm{~A}+$ $25 \% \mathrm{~B}$, and then kept in these conditions for another $5 \mathrm{~min}$ to let column re-equilibration. Flow rate was $1 \mathrm{~mL} \mathrm{~min}^{-1}$, with UV detection at $441 \mathrm{~nm}$. A Nucleosil $120 \mathrm{C} 184.0 \times 150 \mathrm{~mm}$, $5 \mu \mathrm{m}$ particle size, column with a guard column, ODS $4.6 \times 10 \mathrm{~mm}$ (Teknokroma, Barcelona, Spain), was used for reversed phase separation. The chromatographic equipment was a LaChrom Elite ${ }^{\circledR}$ HPLC system from VWR-Hitachi, which was constituted by an L-2130 binary pump equipped with a low pressure gradient accessory and in-line degasser, an L-2420 UV-Vis detector and an L-2200 autosampler. Pigments were quantified as in Montero et al. (2005).

\subsection{Liquid chromatography coupled to mass spectrometry}

The same LaChrom Elite ${ }^{\circledR}$ HPLC system from VWRHitachi and column configurations as above were used for HPLC separation. The gradient elution used for MS analysis was as follows: (1) initial $80 \% \mathrm{~A}+20 \% \mathrm{~B}$, (2) 5 min linear gradient to $15 \% \mathrm{~A}+85 \% \mathrm{~B}$, (3) $8 \mathrm{~min}$ isocratic, (4) $10 \mathrm{~min}$ linear gradient to $0 \% \mathrm{~A}+100 \% \mathrm{~B}$, (5) 16 min isocratic, (6) 20 min linear gradient to $80 \% \mathrm{~A}+20 \% \mathrm{~B}$, (7) 30 min linear gradient to $2 \% \mathrm{~A}+98 \% \mathrm{C},(8) 50 \mathrm{~min}$ isocratic, (9) $60 \mathrm{~min}$ linear gradient to $80 \% \mathrm{~A}$ and $20 \% \mathrm{~B}$, and (10) $75 \mathrm{~min}$ isocratic; where solvent $\mathrm{A}$ is methanol/acenotrile $/ 5 \mathrm{mM}$ aqueous ammonium acetate $(75: 15: 10)$, solvent $\mathrm{B}$ is acetonitrile $/ 5 \mathrm{mM}$ aqueous ammonium acetate (90:10), and solvent $\mathrm{C}$ is $100 \%$ methanol. The flow rate was $0.3 \mathrm{~mL} \mathrm{~min}^{-1}$, and $50 \mu \mathrm{L}$ were injected (Montero 2011).

Column effluent was connected online to the UV detector (L-2420 from Hitachi-VWR), with detection set at $450 \mathrm{~nm}$, and then an ion-trap mass spectrometer (IT-MS) Esquire ${ }^{\circledR}$ 6000 from Bruker Daltonics S.A. (Bruker Daltonik GmbH, Bremen, Germany), which was equipped with an electrospray ionization source (ESI). Compounds were detected in the negative (ESI-) and positive (ESI + ) ion mode in full scan. Mass spectrometer parameters for the analysis were nebulizer $45 \mathrm{psi}$, dry gas $\left(\mathrm{N}_{2}\right) 9.0 \mathrm{Lmin}^{-1}$, dry temperature $320^{\circ} \mathrm{C}$, capillary current $3500 \mathrm{~V}$, scan $50-1200 \mathrm{~m} / \mathrm{z}$, and collision gas was helium. Data were afterwards analysed by using the Data Analysis ${ }^{\circledR}$ software from Bruker Daltonik GmbH (Montero 2011).

\subsection{Statistical analysis}

Data were analysed statistically by using the sofware StatGraphics 5.0. Paired or unpaired tests were conducted according to similarity of variances of each variable. For 
multiple comparison, one-way ANOVA was used. Statistical differences were accepted for $P<0.05$.

\section{Results}

\section{$3.1 \quad H P L C-U V$ analysis of pigments}

As in previous studies, major pigments found in this Synechococcus $\mathrm{sp}$. strain were Chl, Z and Car. Myx exhibited a very low content under LL conditions, but it substantially rose after $48 \mathrm{~h}$ of exposure to HL conditions (see below). The time course of variation in the different pigment content during $\mathrm{HL}$ acclimation is shown in figure 2 at different time intervals. Chl content was substantially reduced by about $40 \%$ in the HL-exposed cultures (figure 2A), whereas it rose in cultures kept under LL conditions after $24 \mathrm{~h}$ and $48 \mathrm{~h}$ of the experiment onset. Statistical analysis showed significant $(P<0.05)$ differences between the Chl contents under $\mathrm{HL}$ at every sampling period except for 0 and $2 \mathrm{~h}$, and 4 and $8 \mathrm{~h}$. The Chl contents under LL were also found to be significantly different to those under HL at equivalent sampling periods (24 and $48 \mathrm{~h}$ ).

Myx content started to rise at about $8 \mathrm{~h}$ of exposure to $\mathrm{HL}$ conditions (figure 2B), but significant differences $(P<0.05)$ with regard to the initial content were only shown for its contents at 24 and $48 \mathrm{~h}$, which rose to about fourfold the initial. Under LL conditions, Myx also exhibited an increase in its content with time in regard to the initial content, but it was lower than under HL conditions, and only after $48 \mathrm{~h}$ its content was significantly $(P<0.05)$ different to the initial one (about 2.8-fold the initial one). The contents of two minor carotenoids that elute a few minutes later than $\mathrm{Z}$ are shown in figure $2 B$ as well. One of these carotenoids $(\lambda=460 \mathrm{~nm})$ has been shown to be hydroxy-echinenone (Graham and Bryant 2009; Montero et al. 2011). Absorption spectrum of the other carotenoid (unkn1) has absorption maxima at 448/ 475 and a spectrum peak III/II ratio of about $40 \%$, which might be indicative of this carotenoid being an intermediate in the $\mathrm{Z}$ or Myx biosynthesis pathway. The content of hydroxy-echinenone dropped after $4 \mathrm{~h}$ of $\mathrm{HL}$ exposure to about $60 \%$ of the initial one. An increase in the content of the second minor carotenoid (unkn1) in relation to the initial one could be only observed after $24 \mathrm{~h}$ of exposure to HL. Appreciable accumulation of any other intermediates of the Myx biosynthesis pathway of those shown in figure 1 could not be detected.

The time course of Car and $\mathrm{Z}$ content evolution is depicted in figure $2 \mathrm{C}$. Initially, the Car content was higher than the $\mathrm{Z}$ content, but this situation reversed just after $8 \mathrm{~h}$ of exposure to HL conditions, which is coincident with the time interval to which the Myx content apparently started to increase. After $24 \mathrm{~h}$ and $48 \mathrm{~h}$ of $\mathrm{HL}$ exposure, the $\mathrm{Z}$ content clearly surpassed the Car content, whereas after $24 \mathrm{~h}$ under
LL conditions Car content still remains above the $\mathrm{Z}$ content. Nonetheless, statistical analysis showed that $\mathrm{Z}$ content was significantly different between the LL and the HL treatment at $48 \mathrm{~h}$ sampling period. Within the HL treatment, $\mathrm{Z}$ content increased significantly after $24 \mathrm{~h}$, from $7.58(\mu \mathrm{mol} / \mathrm{mL}) / \mathrm{A}_{750}$ to $10.60(\mu \mathrm{mol} / \mathrm{mL}) / \mathrm{A}_{750}$. The Car content showed a significant depletion after $4 \mathrm{~h}$ of exposure to $\mathrm{HL}$, with no significant variation afterwards; thus after $48 \mathrm{~h}$ of exposure to $\mathrm{HL}$, the $\mathrm{Car}$ content had decreased by about $3.88(\mu \mathrm{mol} / \mathrm{mL}) / \mathrm{A}_{750}$. In contrast, the Car content under LL conditions showed a significant rise after $24 \mathrm{~h}$ with regard to the initial content, and it was also significantly different with regard to the Car content under HL conditions. The depletion of Car content after $48 \mathrm{~h}$ under $\mathrm{HL}$, which is $3.88(\mu \mathrm{mol} / \mathrm{mL}) / \mathrm{A}_{750}$, was closely equivalent (within error) to the sum of $\mathrm{Z}$ and $\mathrm{Myx}$ content increase, which was $3.68(\mu \mathrm{mol} / \mathrm{mL}) / \mathrm{A}_{750}$.

\subsection{HPLC-MS analysis of lipids}

The different glycerolipid species found in Synechococcus sp. Syn02 were identified by MS/MS data and published literature (Sakamoto et al. 1997; Kim et al. 1999; Montero 2011). Because analysis conditions were the same in both $\mathrm{HL}$ and LL, and cell density was the only parameter that changed from HL to LL, for comparative purposes quantification can be approached within each lipid class by using the peak area normalized to the absorbance measured at $750 \mathrm{~nm}$ $\left(\mathrm{A}_{750}\right)$, this latter being considered as a relative measurement of the cell density for cyanobacterial cells (Sorokin 1973; Montero et al. 2002). The SQDG and MGDG species content dropped in HL-exposed cells as compared to LLexposed cells (table 1). As a general feature, the content of species containing a C18:3 fatty acyl residue rose or remained at the same level. Remarkably, MGDG(18:2/ 16:1), with $m / z 770$ in positive mode, was almost completely depleted while its isobaric species MGDG(18:3/16:0) rose to approximately the same level as MGDG(18:2/16:1) had under LL conditions (figure 3).

Taking these changes into consideration, it was found of interest to determine the time course of the variation observed in those species that experienced an apparently relevant change, which included MGDG(18:2/16:1), MGDG (18:3/16:0), PG(18:3/16:0), PG(18:2/16:0), MGDG(18:3/ $16: 1)$ and DGDG(18:3/16:1). The results of this experiment are illustrated in figure 4b. Depletion of MGDG(18:2/16:1) with concurrent increase in MGDG(18:3/16:0) seems to have occurred after about $8 \mathrm{~h}$ of exposure to HL (figure $4 \mathrm{~b}$ ), when significant differences were statistically shown; however, the sum of these two species content after $48 \mathrm{~h}$ of HL exposure (c.a. $3.91 \times 10^{9}$ ) was lower than the sum of their initial content (c.a. $4.80 \times 10^{9}$ ). No significant differences were found in MGDG(18:3/16:1) content over the $48 \mathrm{~h}$ period, apart from the value at $24 \mathrm{~h}$. $\mathrm{PG}(18: 3 / 16: 0)$ 

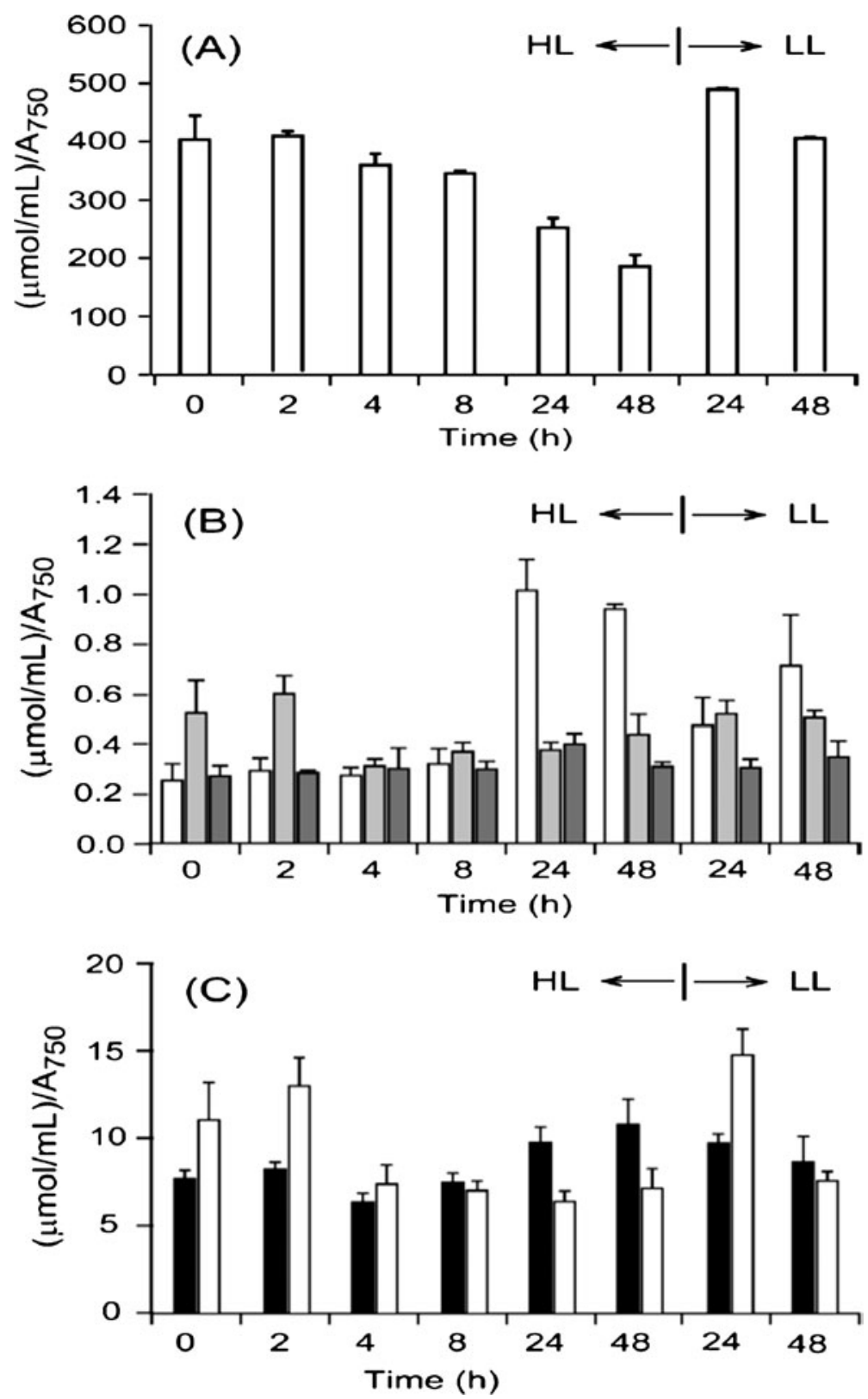

Figure 2. Time course evolution of different pigment content in cultures exposed to high light (HL) and cultures kept in low light (LL) as controls. The content is expressed as $\left((\mu \mathrm{mol}\right.$ of compound $) /(\mathrm{mL}$ of culture $) / \mathrm{A}_{750}$, where $\mathrm{A}_{750}$ is the in vivo absorbance at $750 \mathrm{~nm}$. Panels: (A) chlorophyll $a$; (B) myxoxanthophyll (open bars), hydroxy-echinenone (clear grey bars), and unknown carotenoid (dark grey bars); and (C) zeaxanthin (dark bars), and $\beta$-carotene (open bars).

content rose to about 3.83-fold the initial content only after $48 \mathrm{~h}$ of HL exposure, while PG(18:2/16:0) exhibited a significant content depletion after $24 \mathrm{~h}$ of HL exposure. DGDG (18:3/16:1) content increased to 1.76 -fold the initial content after $48 \mathrm{~h}$ of HL exposure. Reversal of the MGDG(18:2/16:1)/ MGDG(18:3/16:0) ratio to that observed under LL conditions was slow and took more than $72 \mathrm{~h}$ after the cultures were transferred back to LL conditions (data not shown).

\section{Discussion}

\subsection{Pigments}

Even though an apparent rise in Myx under HL conditions seems to have occurred after $8 \mathrm{~h}$, statistically significant differences were only shown for the values at $24 \mathrm{~h}$ and $48 \mathrm{~h}$, while the Car content started to decrease after $4 \mathrm{~h}$; this 
Table 1. Comparative content (mean \pm standard error, $n=3$ ) of each lipid class under high light (HL) and low light (LL), expressed as the sum of the chromatographic peak area (r.u.) of all species and normalized to the in vivo absorbance at $750 \mathrm{~nm}\left(\mathrm{~A}_{750}\right)$, from positive (ESI+) and negative (ESI-) electrospray ionization mass spectrometry

\begin{tabular}{|c|c|c|c|c|c|}
\hline Light & Ionization & SQDG & PG & MGDG & DGDG \\
\hline \multirow[t]{2}{*}{ LL } & ESI+ & $9.67 \pm 0.3810^{9 *}$ & $2.09 \pm 0.7010^{9}$ & $15.00 \pm 0.7610^{9 *}$ & $3.76 \pm 0.9010^{9}$ \\
\hline & ESI- & $9.54 \pm 3.9610^{9}$ & $2.45 \pm 0.8710^{9}$ & $3.82 \pm 0.6710^{9} * *$ & $0.12 \pm 0.0710^{9}$ \\
\hline \multirow[t]{2}{*}{$\mathrm{HL}$} & ESI+ & $5.1 \pm 0.4510^{9} *$ & $2.01 \pm 0.1110^{9}$ & $8.37 \pm 0.6110^{9} *$ & $2.62 \pm 0.0310^{9}$ \\
\hline & ESI- & $4.73 \pm 1.2210^{9}$ & $3.10 \pm 0.4810^{9}$ & $1.54 \pm 0.3610^{9} * *$ & $0.08 \pm<0.0110^{9}$ \\
\hline
\end{tabular}

All other conditions were the same. SQDG: sulfoquinovosyl-diacyl-glycerol, PG: phosphatidyl-diacyl-glycerol, MGDG and DGDG: monoand di-galactosyl-diacyl-glycerol, respectively. The symbols * and ** denote which values calculated from positive or negative ionization are significantly different $(P<0.005)$ within each lipid class.

finding could be indicative of the fact that demand of Lyc (and/or $\gamma$-carotene) for Myx biosynthesis is initiated without an apparent increase in Lyc synthesis occurs. This hypothesis is also underpinned by the delay in $\mathrm{Z}$ content rise, which did not take place until $24 \mathrm{~h}$ of HL exposure as well. Because substantial accumulation of any Myx biosynthetic pathway intermediates could not be detected, apart from that shown for the second minor carotenoid (unkn1), even at very short times after HL exposure (data not shown), together with the long delay required for Myx to show an appreciable increase, it can be suggested that, at least in vivo, the enzymes of the Myx biosynthetic pathway act sequentially, with no limiting steps in any of the intermediates and tightly coupled reactions (Graham and Bryant 2009). Indeed, the intermediates 1-hydroxylycopene, 3-deoxy-myxol (plectaniaxanthin) and myxol have been solely detected in previous studies using mutant strains with any enzyme silenced (Maresca et al. 2007; Graham and Bryant 2009). It may be also considered that demand for Car, which has been shown to be the only carotenoid in PRCs (Loll et al. 2005), is likely reduced because of high light acclimation involves a decrease in PSI units (Murakami and Fujita 1991; Fuhrmann et al. 2009), and hence, a fraction of the Lyc pool would be deviated towards Myx biosynthesis without the need for it being increased. Accordingly, the following event sequence is suggested to occur: (i) within a time interval of $2-4 \mathrm{~h}$ the synthesis of Car is reduced, whose content is likely reduced because of the synthesis of Z, with Lyc being primarily directed towards the Myx biosynthesis pathway; meanwhile, $\mathrm{Chl}$ is degraded and its biosynthesis substantially reduced; (ii) after about $8 \mathrm{~h}$ of HL exposure the activity of the $\beta$ carotene hydroxylase for $Z$ synthesis is moderately enhanced again, which leads to the rise in $\mathrm{Z}$ content to start, whereas Car synthesis remains in a steady state; concurrently, the activity of the enzymes of the Myx pathway is also enhanced; and (iii) after $24 \mathrm{~h}$ of HL exposure a steady state activity of enzymes involved in Car, $\mathrm{Z}$ and Myx biosynthesis is attained, and the relative proportion of these carotenoids is maintained.

\subsection{Membrane lipids}

The lipid configuration in which $\mathrm{C} 18: 3$ and $\mathrm{C} 16: 0$ fatty acyls esterify the glycerol backbone seems to be the predominant one under HL conditions in this Synechococcus strain. Indeed, the SQDG, PG and MGDG species with the above configuration exhibited a substantial increase in their contents, whereas other species were almost depleted. Only MGDG(18:3:/16:1) and DGDG(18:3/16:1) contents did not show a significant modification during light acclimation. Therefore, it may be suggested that C18:3 acyl chains bound to the $s n-1$ position of the glycerol backbone could have significance in regard to glycerolipid functionality. In a similar sense, the physiological relevance of $\mathrm{C} 16$ acyl chains at the $s n-2$ position of the glycerol backbone was shown by Okazaki et al. (2006). According to the present knowledge, this change in fatty acyl composition of the glycerolipid species towards species with more unsaturated acyl chains is likely to have been motivated by the need of higher membrane fluidity, which may be triggered by the HLinduced enhancement of photosynthetic activity, i.e. $\mathrm{O}_{2}$ release, plastoquinone diffusion and PSII repair cycle.

Functional roles of lipids in photosynthetic membranes, in addition to structural roles, are now being outlined by recent experimentation, and in particular for PG (Frentzen 2004; Lee 2004; Jones 2007; Hölzl and Dörmann 2007; Wada and Murata 2007; Sato and Wada 2009). The results obtained in the present study suggest that within PG species, only PG (18:3/16:0) seems to be the most relevant species in membrane configuration for an efficient photosynthesis performance under HL conditions. These changes in PG species could be also related to the concomitant increase in myxoxanthophyll that was observed, as pointed out by Domonkos et al. (2009).

MGDG is the most abundant lipid class in cyanobacterial membranes (Hölzl and Dörmann 2007; Sato and Wada 2009). In this study, MGDG species were shown to undergo a significant reduction in their total content under HL conditions in relation to LL conditions (table 1). Since MGDG 

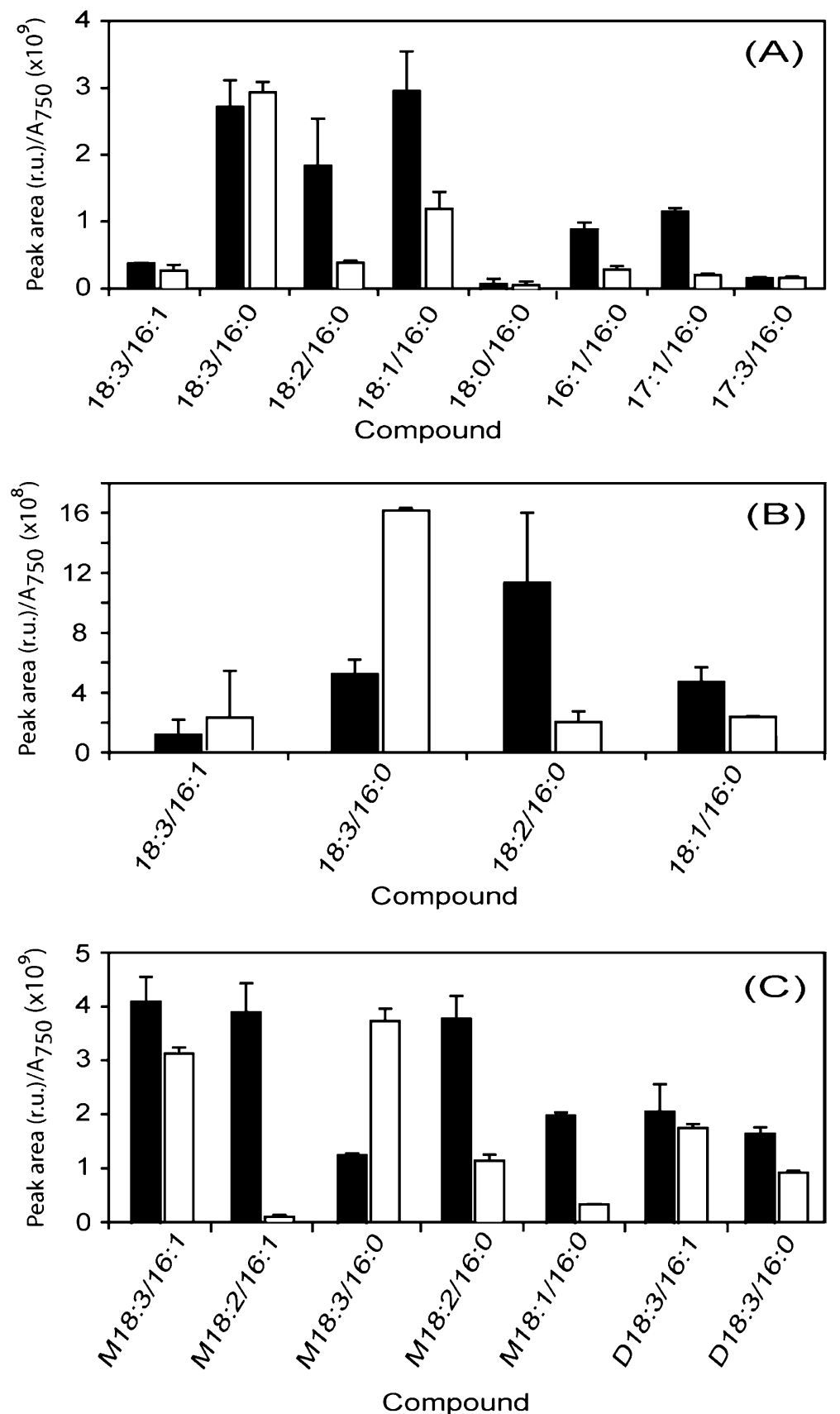

Figure 3. Comparative content from positive ionization of the different membrane lipid species, expressed as the chromatographic peak area and normalized to the in vivo absorbance at $750 \mathrm{~nm}\left(\mathrm{~A}_{750}\right)$. High light: lopen bars, and low light: dark bars. Panels: (A) SQDG, (B) PG, and (C) MGDG and DGDG (M and D in X-axes mean MGDG and DGDG, respectively).

species are known to be located on both leaflets of the thylakoid membrane (Guskov et al. 2009; Kern et al. 2009), whose lipids account for up to $90 \%$ of the cyanobacterial membrane lipids (Murata et al. 1981), the reduction in the total MGDG content might suggest a decrease in the membrane area, which could in turn be related to the reduction in PSI that has been documented to take place under high light in cyanobacteria (Murakami and Fujita 1991; Fuhrmann et al. 2009). This suggestion would be in accordance with the reduction in $\beta$-carotene and Chla contents indicated above. Furthermore, considering that $\mathrm{PG}$ and SQDG species are known to be located on the stromal side of the thylakoid membrane (Fuhrmann et al. 2009; Guskov et al. 2009), where MGDG species are also present, it may 

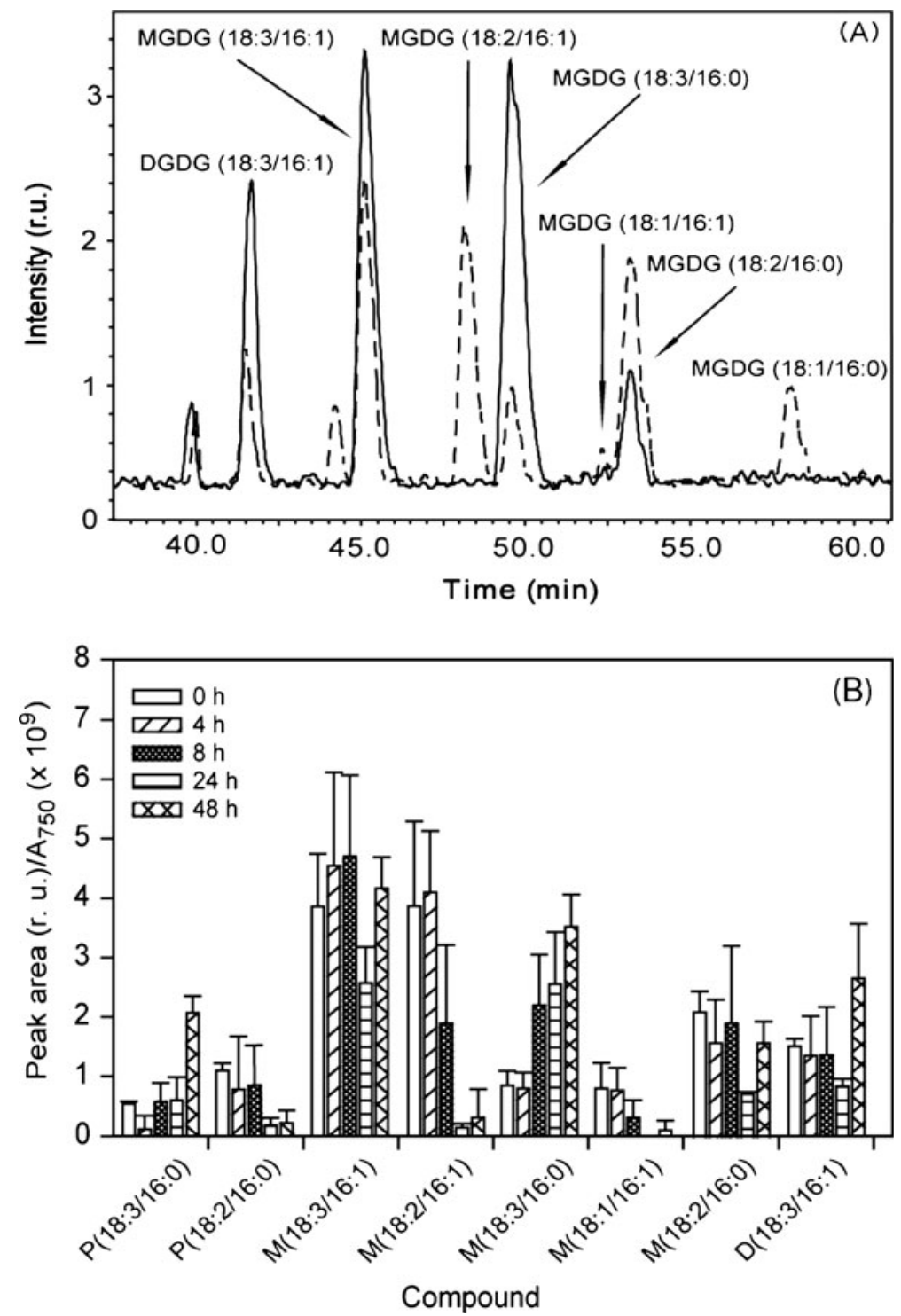

Figure 4. (A) Chromatograms showing the different relative proportion of MGDG(18:3/16:0) and MGDG(18:2/16:1) contents in cells exposed to high light (continuous line) in relation to cells exposed to low light (dashed line). (B) Time course evolution of different lipid species content, expressed as the chromatographic peak area determined from analysis with positive ionization and normalized to the in vivo absorbance at $750 \mathrm{~nm}\left(\mathrm{~A}_{750}\right)$. X-axes: P means PG, and M and D mean MGDG and DGDG, respectively.

be suggested that primary changes in membrane conformation and size elicited by HL conditions are to take place in the membrane leaflet facing its stromal side, with the anionic PG augmenting its content significantly. This feature might contribute to facilitate proton translocation from the lumen to the stroma.

Of particular concern is the depletion of MGDG(18:2/ 16:1) as concomitant rise in MGDG(18:3/16:0) occurred after cells were exposed to HL conditions, although this change in MGDG species may also be envisaged within the general changes discussed above. Two factors are currently described to affect membrane fluidity, which are the geometry of the glycerolipid and the number of double bonds. The content change in these two MGDG species encompasses neither variation in glycerolipid-associated geometry nor double bond number modification, apart from the fact that the three double bonds are located within the same acyl chain in MGDG(18:3/16:0). Therefore, irrespective of these two factors, we find that the only feature that could be relevant in the MGDG configuration change from C18:2/ $\mathrm{C} 16: 1$ to $\mathrm{C} 18: 3 / \mathrm{C} 16: 0$ in regard to membrane properties is that it implies a more linear and hydrophobic residue at the $s n-2$ position and a more bended residue at the $s n-1$ position of the glycerol backbone, with the three double bonds 
supporting an electron-rich nodule. Therefore, we are prone to hypothesize on the fact that this MGDG composition change is to be likely related to an acyl chain-induced effect on the glycerolipid shape, this hypothesis being similar to what Barkan et al. (2006) explained as an unexpected chilling-resistance phenotype after the replacement of C16:3 in MGDG by the saturated acyl group C16:0 in the fad5-2 mutant of Arabidopsis plants. Hence, it is likely that glycerolipid functionality regarding membrane fluidity and photosynthetic component diffusion is dependent not only on the head group but also on the particular acyl chains (Mullineaux 2008). Indeed, changes in the shape of lipid molecules have been reported to be of importance for different membrane functions (Gounaris and Barber 1983; Simidjiev et al. 2000). Contrary to other membrane lipid classes, MGDG is a non-bilayer forming glycerolipid because of its 'cone-like' geometry (Gounaris and Barber 1983). Diverse authors have shown the importance of nonbilayer lipids for full protein functionality (Simidjiev et al. 2000; de Kruijff 1997; Garab et al. 2000). This feature has been attributed to the high lateral pressure exerted by the acyl groups of the non-bilayer lipids on the proteins embedded in the membrane, which could contribute to keep the protein subunits in the proper arrangement. It could then be hypothesized on the highly bended C18:3 acyl chain being the responsible for such lateral pressure because of the alternating three double bonds acting like a 'stress tensor', while the linear C16:0 acyl chain acts like a 'nail' and functions in keeping the lipid embedded within the bilayer. Nonetheless, hypotheses drawn above are devoted of experimental testing.

Regarding the time course of variation in both pigment and membrane lipid contents, it is noteworthy that major changes seem to have been accomplished in a time interval of about $24 \mathrm{~h}$, which has also been reported to be the time necessary for phycobilisome degradation (DeRuyter and Fromme 2008). Hence, it may be suggested that the reorganization of the photosynthetic apparatus is a coordinated process, which simultaneously encompasses both protein embedding into the membrane and lipid species modification, with specific lipid species contributing to the targeting signal for protein sorting after translocation (Spence et al. 2003).

\section{Acknowledgments}

Financial support from the Spanish Ministry of Education and Science (project reference AGL2004-08215-C03-02) is greatly appreciated.

\section{References}

Awai K, Watanabe H, Benning C and Nishida I 2007 Digalactosyldiacylglycerol is required for better photosynthetic growth of
Synechocystis sp. PCC6803 under phosphate limitation. Plant Cell Physiol. 48 1517-1523

Barkan L, Vijayan P, Carlsson AS, Mekhedov S and Browse J 2006 A suppressor of fabl challenges hypotheses on the role of thylakoid unsaturation in photosynthetic function. Plant Physiol. 141 1012-1020

de Kruijff B 1997 Lipids beyond the bilayer. Nature 386 129-130

DeRuyter YS and Fromme P 2008 Molecular structure of the Photosynthetic Apparatus; in The Cyanobacteria. Molecular biology, genomics and evolution (eds) A Herrero and E Flores (Caister Academic Press, Norfolk, UK) pp 217-269

Domonkos I, Malec P, Sallai A, Kovács L, Itoh K, Shen G, Ughy B, Bogos B, Sakurai B, Kis M, Strzalka K, Wada H, Itho S, Farkas $\mathrm{T}$ and Gombos Z 2004 Phosphatidylglycerol is essential for oligomerization of photosystem I reaction center. Plant Physiol. 134 1471-1478

Domonkos I, Malec P, Laczko-Dobos H, Sozer O, Klodawska K, Wada H, Strzalka K and Gombos Z 2009 Phosphatidylglycerol depletion induces an increase in myxoxanthophyll biosynthetic activity in Synechocystis PCC6803 cells. Plant Cell Physiol. 50 373-382

Dörmann P and Benning C 2002 Galactolipids rule in seed plants. Trends Plant Sci. 7 112-118

Falkowsky PG and Raven JA 1997 Aquatic Photosynthesis (Malden, Massachusetts: Blackwell Science)

Frentzen M 2004 Phosphatidylglycerol and sulfoquinovosyldiacylglycerol: anionic membrane lipids and phosphate regulation. Curr. Opin. Plant Biol. 7 270-276

Fuhrmann E, Gathmann S, Rupprecht E, Golecki J and Schneider D 2009 Thylakoid membrane reduction affects the photosystem stoichiometry in the cyanobacterium Synechocystis sp. PCC 6803. Plant Physiol. $149735-744$

Garab G, Lohner K, Laggner P and Farkas T 2000 Self-regulation of the lipid content of membranes by non-bilayer lipids: a hypothesis. Trends Plant Sci. 5 489-494

Gounaris K and Barber J 1983 Monogalactosyldiacylglycerol: the most abundant polar lipid in nature. Trends Biochem. Sci. 8 378-381

Graham JE and Bryant DA 2008 The biosynthetic pathway for synechoxanthin, an aromatic carotenoid synthesized by the euryhaline, unicellular cyanobacterium Synechococcus sp. strain PCC7002. J. Bacteriol. 190 7966-7974

Graham JE and Bryant DA 2009 The biosynthetic pathway for myxol-2' fucoside (myxoxanthophyll) in the cyanobacterium Synechococcus sp. strain PCC7002. J. Bacteriol. 191 3292-3300

Grzyb J, Gagos M, Gruszecki WI, Bojko M and Strzalka M 2008 Interaction of ferredoxin:NADP ${ }^{+}$oxidoreductase with model membranes. Biochim. Biophys. Acta 1778 132-143

Güler S, Seeliger A, Härtel H, Renger G and Benning C 1996 A null mutant of Synechococcus sp. PCC7942 deficient in the sulfolipid sulfoquinovosyl diacylglycerol. J. Biol. Chem. 271 7501-7507

Guskov A, Kern J, Gabdulkhakov A, Broser M, Zouni A and Saenger W 2009 Cyanobacterial photosystem II at 2.9- $\AA$ resolution and the role of quinones, lipids, channel and chloride. Nature Struct. Mol. Biol. 16 334-342.

Hirschberg J and Chamovitz D 1994 Carotenoids in cyanobacteria; in The molecular biology of cyanobacteria (ed.) DA Bryant (Dordrecht: Kluwer Academic Publishers) pp 559-579 
Hölzl G and Dörmann P 2007 Structure and function of glycoglycerolipids in plants and bacteria. Prog. Lipid Res. 46 225-243

Jones MR 2007 Lipids in photosynthetic reaction centres: structural roles and functional holes. Prog. Lipid Res. 46 56-87

Kern J, Zouni A, Guskov A and Krau $\beta$ N 2009 Lipids in the structure of Photosystem I, Photosystem II and the Cytochrome $b_{6} f$ Complex; in Lipids in photosynthesis: essential and regulatory functions (eds) H Wada and N Murata (Springer Science + Business Media BV) pp 203-242

Kim YH, Choi J-S, Yoo JS, Park Y-M and Kim MS 1999 Structural identification of glycerolipid molecular species isolated from cyanobacterium Synechocystis sp. PCC 6803 using Fast Atom Bombardment Tandem Mass Spectrometry. Anal. Biochem. 267 260-270

Kobayashi K, Awai K, Nokamura M, Nagatani A, Masuda T and Ohta H 2009 Type-B monogalactosyldiacylglycerol synthases are involved in phosphate starvation-induced lipid remodeling, and are crucial for low phosphate adaptation. Plant J. 57 322-331

Lagarde D and Vermaas W 1999 The zeaxanthin biosynthesis enzyme $\beta$-carotene hydroxylase is involved in myxoxanthophyll synthesis in Synechocystis sp. PCC6803. FEBS Lett. 454 247251

Lee AG 2004 How lipids affect the activities of integral membrane proteins. Biochim. Biophys. Acta 1666 62-87

Leng J, Sakurai I, Wada H and Shen J-R 2008 Effects of phospholipase and lipase treatments on photosystem II core dimer from a thermophylic cyanobacterium. Photosyn. Res. 98 469-478

Loll B, Kern J, Saenger W, Zouni A and Biesiadka J 2005 Towards complete cofactor arrangement in the $3.0 \AA$ resolution structure of photosystem II. Nature 438 1040-1044

Maresca JA, Graham JE, Wu M, Eisen JA and Bryant DA 2007 Identification of a fourth family of lycopene cyclases in photosynthetic bacteria. Proc. Natl. Acad. Sci. USA 104 11784-11789

Masamoto K and Furukawa KI 1997 Accumulation of zeaxanthin in cells of the cyanobacterium, Synechococcus sp. strain PCC 7942 grown under high irradiance. J. Plant Physiol. 151 257-261

McIntosh TJ and Simon SA 2006 Roles of bilayer material properties in function and distribution of membrane proteins. Ann. Rev. Biophys. Biomol. Struct. 35 177-198

McIntyre HL, Kana TM, Anning T and Geider RJ 2002 Photoacclimation of photosynthesis response curves and photosynthetic pigments in microalgae and cyanobacteria. J. Phycol. 38 17-38

Mohamed HE, van de Meene AML, Roberson RW and Vermaas WFJ 2005 Myxoxanthophyll is required for normal cell wall structure and thylakoid organization in the cyanobacterium Synechocystis sp strain PCC6803. J. Bacteriol. 187 6883-6892

Montero O 2011 Lipid Profiling of Synechococcus sp. PCC7002 and Two Related Strains by HPLC Coupled to ESI-(Ion Trap)MS/MS. Z. Naturforsch. 66c 149-158

Montero O, Klisch M, Häder D-P and Lubián LM 2002 Comparative sensitivity of seven marine microalgae to cumulative exposure to ultraviolet-B radiation with daily increasing doses. Bot. Mar. 45 305-315

Montero O, Macías-Sánchez MD, Lama CM, Lubián LM, Mantell C, Rodríguez M, de la Ossa EM 2005 Supercritical $\mathrm{CO}_{2}$ extraction of $\beta$-carotene from a marine strain of the Cyanobacterium Synechococcus species. J. Agric. Food Chem. 53 9701-9707

Montero O, Porta JM, Porta J, Martínez G and Lubián LM 2011 Characterization of two Synechococcus sp. PCC7002-related cyanobacterial strains in relation to $16 \mathrm{~S}$ rDNA, crtR gene, lipids and pigments. Phycol. Res. 59 147-155

Mullineaux CW 2008 Biogenesis and dynamics of thylakoid membranes and the photosynthetic apparatus; in The Cyanobacteria. Molecular biology, genomics and evolution (eds) A Herrero and E Flores (Caister Academic Press, Norfolk, UK) pp 289-303

Murakami A and Fujita Y 1991 Regulation of photosystem stoichiometry in the photosynthetic system of the cyanophyte Synechocystis PCC 6714 in response to light intensity. Plant Cell Physiol. 32 223-230

Murata N, Sato N, Omata T and Kuwabara T 1981 Separation and characterization of thylakoid and cell-envelope of the bluegreen-alga (cyanobacterium) Anacystis nidulans. Plant Cell Physiol. 22 403-410

Okazaki Y, Sato N, Tsuji N, Tsuzuki M and Nishida I 2006 The significance of $\mathrm{C} 16$ fatty acids in the $s n-2$ positions of glycerolipids in the photosynthetic growth of Synechocystis sp. PCC6803. Plant Physiol. 141 546-556

Okazaki Y, Shimojima M, Sawada Y, Toyooka K, Narisawa T, Keiichi M, Tanaka H, Matsuda F, Hirai A, Hirai MY, Ohta H and Saito K 2009 A chloroplastic UDP-Glucose pyrophosphorylase from Arabidopsis is the committed enzyme for the first step of sulfolipid biosynthesis. Plant Cell 21 892-909

Palsdottir H and Hunte C 2004 Lipids in membrane protein structures. Biochim. Biophys. Acta 1666 2-18

Riethman H, Bullerjahn G, Reddy KJ and Sherman LA 1988 Regulation of cyanobacterial pigment-protein composition and organization by environmental factors. Photosyn. Res. 18 133-161

Sakamoto T, Higashi S, Wada H, Murata N and Bryant DA 1997 Low-temperture-induced desaturation of fatty acids and expression of desaturase genes in the cyanobacterium Synechococcus sp. PCC 7002. FEMS Microbiol. Lett. 152 313-320

Sato N 2004 Roles of the acidic lipids sulfoquinovosyl diacylglycerol and phosphatidylglycerol in photosynthesis: their specificity and evolution. J. Plant Res. 117 495-505

Sato N and Wada H 2009 Lipid biosynthesis and its regulation in cyanobacteria; in Lipids in photosynthesis: essential and regulatory functions (eds) H Wada and N Murata (Springer Science+ Business Media BV) pp 157-177

Sato NR, Hagio M, Wada H and Tsuzuki M 2000 Requirement of phosphatidylglycerol for photosynthetic function in thylakoid membranes. Proc. Natl. Acad. Sci. USA 97 10655-10660

Schäfer L, Vioque A and Sandmann G 2005 Functional in situ evaluation of photosynthesis-protecting carotenoids in mutants of the cyanobacterium Synechocystis PCC6803. J. Photochem. Photobiol. B: Biol. 78 195-201

Schagerl M and Müller B 2006 Acclimation of chlorophyll $a$ and carotenoids levels to different irradiances in four freshwater cyanobacteria. J. Plant Physiol. 163 709-716

Simidjiev I, Stoylova S, Amenitsch H, Jávorfi T, Mustárdy L, Laggner P, Holzenburg A and Garab G 2000 Self-assembly of large, ordered lamellae from non-bilayer lipids and integral 
membrane proteins in vitro. Proc. Natl. Acad. Sci. USA 97 $1473-1476$

Sorokin C 1973 Dry weight, packed cell volume and optical density; in Handbook of physiological methods (ed) JR Stein (Cambridge University Press, Cambridge, UK) pp 321-344

Spence E, Sarcina M, Ray N, Moller SG, Mullineaux CW and Robinson C 2003 Membrane-specific targeting of green fluorescent protein by the Tat pathway in the cyanobacterium Synechocystis PCC6803. Mol. Microbiol. 48 1481-1489.

Steiger S, Schäfer L and Sandmann G 1999 High-light-dependent upregulation of carotenoids and their antioxidative properties in the cyanobacterium Synechocystis PCC6803. J. Photochem. Photobiol. B: Biol. 52 14-18

Wada H and Murata N 2007 The essential role of phosphatidylglycerol in photosynthesis. Photosyn. Res. 92 205-215
Welti R, Wang X and Williams TD 2003 Electrospray ionization tandem mass spectrometry scan modes for plant chloroplast lipids. Anal. Biochem. 314 149-152

Yao H, Shi Y, Gao R, Zhang G, Zhang R, Zheng C and Xu B 2006 Isolation of lipids from photosystem I complex and its characterization with high performance liquid chromatography/electrospray ionization mass spectrometry. J. Chromatogr. B 837 101-107

Zepke HD, Heinz E, Radunz A, Linscheid M and Pesch R 1978 Combination and positional distribution of fatty acids in lipids from blue-green algae. Arch. Microbiol. 119 157-162

Zhou F, Liu S, Hu Z, Kuang T, Paulsen H and Yang C 2009 Effect of monogalactosyldiacylglycerol on the interaction between photosystem II core complex and its antenna complexes in liposomes of thylakoid lipids. Photosyn. Res. 99 185-193

MS received 02 March 2012; accepted 28 May 2012

Corresponding editor: BASUTHKAR J RAO 